Article

\title{
Integrating Social Justice and Political Engagement into Engineering
}

\author{
Skye Niles ${ }^{1}$, Shawhin Roudbari ${ }^{2}$, and Santina Contreras ${ }^{3}$ \\ ${ }^{1}$ University of Colorado at Boulder, niles.skye@colorado.edu \\ ${ }^{2}$ University of Colorado at Boulder, shawhin@colorado.edu \\ ${ }^{3}$ The Ohio State University, contreras.78@,osu.edu
}

Many engineering activists have emphasized the need to reframe engineering as a sociotechnical field in order to expand engineers' contributions to social justice and peace. Yet, reframing engineering as sociotechnical does not always lead to critical engagement with social justice. We provide several examples of how "social" aspects have been brought into engineering in a depoliticized manner that limits engagement with political and social justice goals. We link these examples to Cech's three pillars of the "culture of disengagement" in engineering:

social/technical dualisms, meritocracy, and depoliticization. We argue that reframing engineering as sociotechnical addresses the first pillar, the social/technical dualism, but does not necessarily include the second and third pillars. We propose that all three pillars can be addressed through integrating explicit attention to political engagement and social justice in efforts to reframe engineering as a sociotechnical field. Doing so can increase engineers' capacity to contribute to social justice and peace.

\section{Sociotechnical Frameworks in Engineering}

Many engineering activists and scholars have drawn attention to the need to reframe engineering as a heterogeneous, sociotechnical field (Cech 2014; Cumming-Potvin and Currie 2013; Faulkner 2007; Leydens and Lucena 2018; Riley 2008; Winner 1980). These scholars critique dominant concepts of engineering practice as a technical endeavor, devoid of social context and consequences. Reframing engineering as sociotechnical disrupts the existing borders and boundaries of engineering. Broadening engineering practice beyond technical problem-solving opens up engineering to a more complex engagement with society-technology relations, and increases the potential of engineers to contribute to a more just and peaceful world. This more expansive and critical engineering practice invites consideration of questions such as what kinds of values are embedded in technologies; who benefits and who suffers the costs of technologies; 
and how are projects and ideas related to capitalism, industry, the military, and the environment (Cech 2014; Riley 2008).

Framing engineering as a sociotechnical practice and considering how society and technology are co-constructed enables engineers to think more critically and act more ethically. For example, rather than solely focusing on safety issues in ethical design, by adopting a broader understanding of how technology interacts with society, engineers can widen their ethical standpoint to consider not only what is safe, but how risks, costs, and benefits are distributed in society (Bucciarelli 2008; Cech 2014; Conlon 2008; Herkert 2000; Lynch and Klein 2000). Rather than considering technically complex engineering as "good" engineering, explicit attention to social issues could shift what counts as "good" engineering towards practices that foster public good and create more equitable social conditions (Harris 2013).

Several scholars have also argued that an increased emphasis on sociotechnical interactions in engineering may increase diversity in the field (Adams et al. 2011; Faulkner 2007; Litchfield and Javernick-Will 2015; Riley 2008). By bringing attention to issues related to social inequality in engineering and by increasing reflexivity within engineering about the ways that certain people, values, and viewpoints may be marginalized, these authors argue that this creates a more inclusive and expansive profession where a variety of people can participate and feel as though they belong. Developing a sociotechnical understanding and practice of engineering can therefore improve equitable experiences both internally to the field and externally in society.

In this paper, we provide examples of how engineering programs and scholars have attempted to shift the focus of engineering away from a technocentric field towards a more sociotechnical framework, namely through a cultural shift in the emphasis in engineering to bring renewed attention to existing sociotechnical practices, and by a structural shift in the content of engineering education coursework to include increased explicit engagement with societytechnology interactions. Indeed, there are important sociotechnical shifts in borders and boundaries of engineering towards more humanitarian initiatives, a greater emphasis on societytechnology relationships, increased attention to STEAM (Science, Technology, Engineering, Arts, and Math) and the value of arts in engineering and other sciences, as well as movements to emphasize gender, racial, and ethnic diversity.

However, despite the broad vision of many engineering scholars and activists to create transformative impacts within engineering through sociotechnical framings of the field-for example, increasing diversity, improving ethical conduct, critiquing capitalism and consumerism, and protesting the salience of extractive and military industries in engineering - we find that many of these impacts fall short of their transformative ideals. A look through the American Society of Engineering Education's daily news feed demonstrates that the vast majority of articles do not explicitly address issues of social justice, criticisms of capitalism, or critiques of the military or industrial influences in engineering; however, there are some appeals to increasing diversity and innovation (ASEE 2018). We find that developing a sociotechnical framing of engineering may lead to increased diversity, improved ethics, and pro-social actions, but it does not necessarily do so. This inconsistency prompts a further exploration of what factors may stall 
more widespread transformations towards social justice, peace, and public welfare engagement in engineering.

\section{Addressing the Three Pillars of Disengagement}

Cech (2014) has argued that the exclusion of public welfare considerations in engineering stem from three ideological pillars: the technical/social dualism within engineering (the hierarchical separation of technical and social concerns), a culture of individual meritocracy (the belief that success is derived from individual efforts, disconnected from systems of privilege and disadvantage), and the ideology of depoliticization (the conviction that engineering can and should be detached from considerations of power and social justice). Drawing from these findings, we argue that addressing one of these pillars without the others is not enough to achieve widespread transformations in engineering culture and practice. Namely, we advocate for the need to address the other two pillars that Cech identifies - depoliticization and individual meritocracy - as a part of sociotechnical framings of the engineering field. We propose that "the social" aspects of sociotechnical frameworks should be engaged with more explicitly in order to achieve more transformative goals. This entails asking, what is meant by "the social," what does it include or exclude, and what values are embedded within it?

One way of achieving more specific engagement with "the social" is to integrate an explicit inclusion of political engagement and social justice throughout the engineering field. Political engagement addresses the ideological pillar of depoliticization by drawing attention to how power inequalities shape the engineering profession and practice-including questions regarding what kind of knowledge is valued, how problems and solutions are conceptualized, whose views are prioritized and valued, and who profits from or is harmed by engineering projects. Integrating considerations of social justice addresses the ideology of individual meritocracy by focusing on how social conditions are shaped by complex systems of privilege and oppression, rather than individual efforts or character. By focusing on all three pillars, engineers can approach their work by considering how social context and power inequalities interact with technology in order to promote, detract from, and define public good.

Below, we describe some of the ways that sociotechnical frameworks have been applied to engineering, with specific regard to efforts to increase diversity in the engineering profession and changes in the content of engineering education. Then, we identify some limitations of addressing only the social/technical pillar of engineering ideology. Next, we propose how all three ideological pillars may be addressed through explicit political engagement and focus on social justice in sociotechnical frameworks of engineering. We conclude with reflections on the possibilities that explicit attention to political engagement and social justice can bring to the engineering profession and to society.

\section{Reframing Engineering as Sociotechnical: Emphasis- and Content-Based Approaches}

Framing is a key political process of meaning-making and boundary work that social movement actors use to accomplish their goals. As Benford and Snow (2000) describe, framing is a dynamic social process through which political or social movement groups characterize the issues that they seek to address, identify solutions, and motivate people to act to accomplish these goals. Framing 
can involve amplification of existing values as well as extension of values to encompass new terrain (Benford and Snow 2000). Reframing engineering as sociotechnical has been approached in both of these ways: through amplifying certain existing values and content (emphasis-based approaches), and also through extending values and content to expand what or who is included within engineering (content-based approaches).

Emphasis-based approaches critique how dominant conceptions of engineering emphasize some aspects of engineering, such as mathematical skills, and marginalize others, such as social context, even though both are necessary parts of engineering practice. For example, Faulkner (2007) argues that despite the dominant ideology of technocentrism, in actual practice engineering is quite heterogeneous and involves a variety of non-technical skills, such as collaboration and communication. Emphasis-based critiques may challenge dominant narratives about why someone should become an engineer, such as being "good at math and science," and advocate for more emphasis on other skills, such as creativity and teamwork, which are also core (though discursively marginalized) aspects of engineering. These emphasis-based approaches reframe engineering by highlighting aspects of engineering that have always existed, but that have been marginalized in dominant conceptions of engineering.

In regard to diversity within engineering, this emphasis-based approach may involve bringing attention to the presence of women, people of color, people with different abilities, and people of different socio-economic backgrounds within engineering in order to emphasize existing diversity within the engineering profession. One example of this is the "\#ILookLikeAnEngineer" campaign. This campaign addresses stereotypes about who is an engineer, and highlights the wide diversity of people practicing engineering (Lien 2015). Increasing the visibility of people other than white, male engineers can alter conceptions about what kinds of people comprise the engineering field.

Another approach to reframing engineering as sociotechnical involves a content-based approach. Content-based approaches entail bringing in new content into engineering, merging disciplinary boundaries, and expanding the boundaries of engineering to more explicitly focus on social-technical interactions. One example of this is an increased interested in "STEAM" or Science, Technology, Engineering, Arts, and Math - which involves the integration of arts and an increased emphasis on creativity, values, and morals in engineering programs (Lachman 2018). Another example is the emergence of humanitarian engineering programs in many campuses across the United States (Nieusma and Riley 2010). Through these programs, the content of engineering programs has been altered to include focus on development studies, economics, and global health, for example. These programs alter what is included and excluded within the boundaries of engineering knowledge to include an explicit emphasis on the social context and social impacts of engineering. Regarding diversity within engineering, these content-based types of approaches may also involve structural changes towards increasing scholarships or funding streams to support underrepresented students within engineering, or changing course content to include material that represent issues that are relevant to a more diverse spectrum of students.

Both the emphasis-based and content-based approaches provide important contributions to reframing engineering as sociotechnical, increasing diverse representation of students within 
engineering, and improving the ability of engineers to work in ways that more equitably serve society. The two approaches also overlap in some instances - for example, STEAM initiatives may not only add new content into engineering programs, but may also involve a new emphasis on the kinds of qualities that are valued in engineering (e.g., collaboration, listening, patience, creativity). However, both of these approaches have limitations in the ways that they can improve equitable experiences within engineering education, as well as broadening the impact of engineering practice on public welfare, an issue that we explore next.

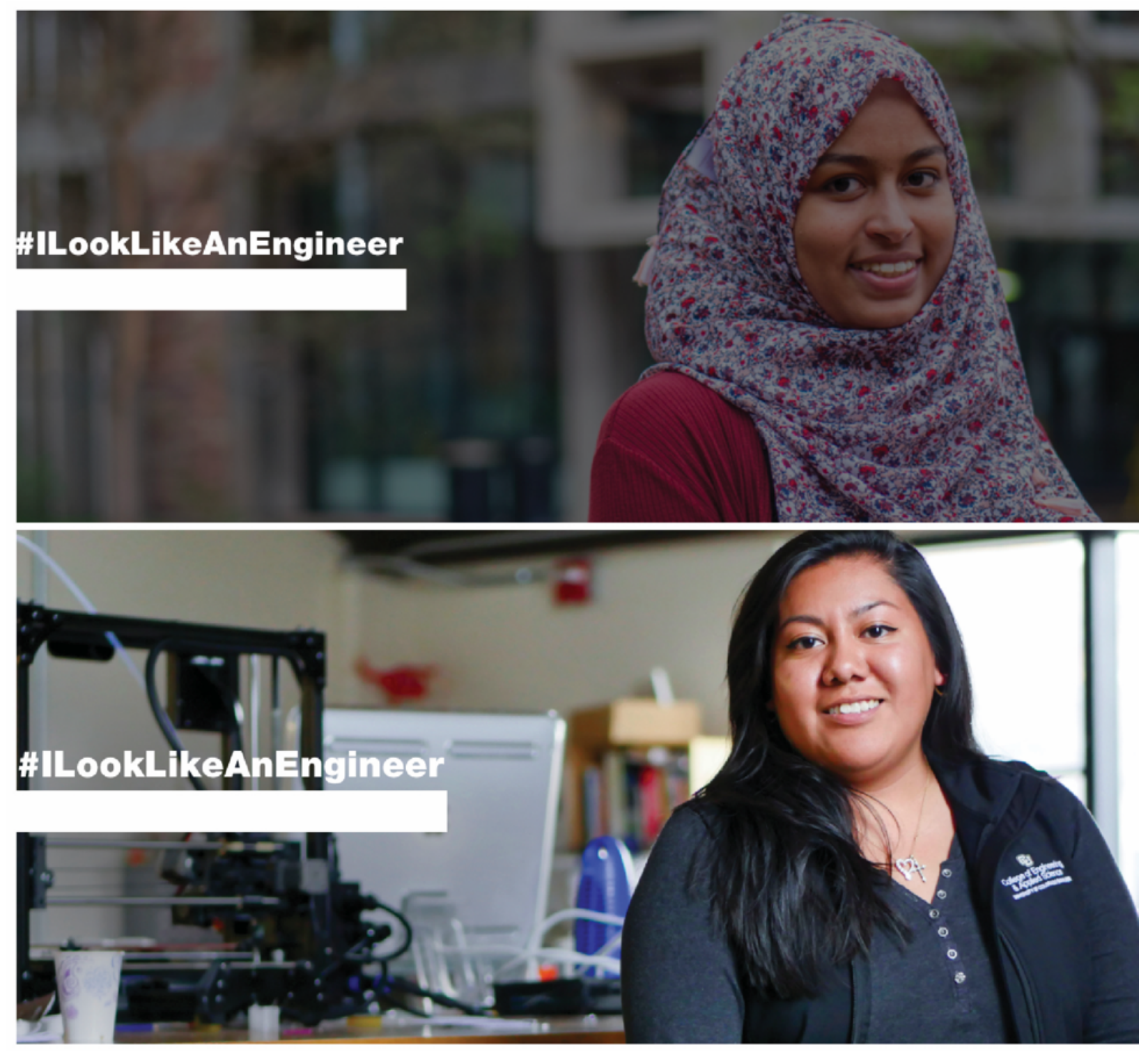

The "\#ILookLikeAnEngineer" campaign has developed through Twitter, professional organizations, and college campuses. Above are pictures from the University of Colorado Boulder \#ILookLikeAnEngineer" website (https://www.colorado.edu/engineering/ilook).

\section{Limitations of Dominant Sociotechnical Frameworks}


These two approaches to sociotechnical reframing make important, yet incomplete, contributions to improving public welfare, social justice, and diversity in the engineering profession and practice. An emphasis-based approach to reframing engineering is limited in its ability to transform more systemic issues that lead to inequality within engineering or encompass new content within the engineering field. Notably, despite dominant technocentric framings, engineering is heterogeneous and involves a diverse array of non-technical skills and considerations (Faulkner 2007). However, this argument alone does not necessarily lead to the transformation of the system that has disregarded those aspects of engineering thus far, nor does it necessarily translate into improving public welfare or social justice concerns.

Recognizing that there is a diverse range of people who are engineers-women, people of color, and people with disabilities, for example — is not the same as questioning and addressing the systems within engineering and society that marginalize these groups of people. To say that women exist in engineering is not the same as ensuring that women have equitable experiences in engineering or asking how the engineering profession reproduces gender inequalities. To point out that some engineers are people of color does not explicitly critique the kinds of interpersonal and systemic discrimination they may face, or question how their experiences within engineering may be different than those of white engineers.

Therefore, the impact of these emphasis-based movements to shift dominant narratives about engineers and engineering are constrained by the spectrum of existing practices and ideologies within the engineering field. It is possible to shift the focus from one aspect of engineering to another, but this does not necessarily lead to the transformation of discriminatory aspects of the entire engineering system - whether these systems are discriminatory in their valuation of certain engineering skills or others, or whether their structured and implicit biases favor certain gender, racial, and ethnic groups.

Content-based approaches are also limited because increasing program content about social context or about engineering for humanitarian causes will not necessarily lead to equitable cultural transformations within the engineering field itself, nor do these efforts inevitably reduce social inequalities in society more broadly. For example, humanitarian engineering initiatives may involve an increased attention to poverty and inequality and broaden engineering education content to consider global inequalities. Yet, these endeavors may also be critiqued for their complicity in "white savior" narratives that reproduce racial hierarchies (Cole 2012). In U.S.based humanitarian engineering programs, when students learn to think about engineering as a means to improve social "good," they also receive messages that they, as Western engineers, have a responsibility to bring this "good" to others. This implicitly inscribes hierarchies between engineers (knower/maker/giver) and communities (receivers), which are often located in the Global South or in economically disadvantaged communities of color in the United States. Humanitarian programs often remain remarkably apolitical despite the striking power dynamics between the Global North and Global South and between scientific expertise and lay knowledge (Nieusma and Riley 2010). Therefore, the inclusion of new types of content within engineering programs - without a critique of power dynamics within these programs and society-does not necessarily lead to more equitable practices or socially just outcomes. 
Furthermore, efforts to increase diversity within engineering through additional outreach and funding without a critique of the kinds of cultural norms and practices that create an exclusionary culture in engineering does not inevitably result in greater equity within engineering. Incentivizing underrepresented students to join engineering programs may be in some ways punitive, rather than supportive, if these students are being brought into hostile climate. And if some of the reasons for increasing and emphasizing diversity are to improve the marketability of an engineering program or company, or expand the profit attained from a product by making it applicable to a more diverse spectrum of users (the "diversity is good for business" argument), these efforts to increase diversity may undermine themselves by strengthening a capitalist, extractive logic that diversity must be linked to economic gain, rather than equity and social justice.

Leong (2013) has documented how universities have gone so far as to use Photoshop to insert photographs of people of color into public relations documents, such as university recruitment brochures, in order to promote the image of racial inclusiveness. In this way, predominantly white institutions (such as universities) gain value and legitimacy from showcasing diversity, but they do so in a way that misrepresents the actual racial composition of the institution. This veneer of diversity is damaging in that it allows universities to display an illusion of racial inclusion without necessarily engaging in the painstaking efforts necessary to meaningfully achieve racial equity (Leong 2013). The superficial display of diversity can forestall social justice efforts if it is not linked to broader institutional change and engagement with historically entrenched systems of racial, gender, and class privilege and oppression.

\section{Missing Links: Political Engagement and Social Justice in Engineering}

The limitations we describe above are linked through the apoliticism present in how each approach can be applied, including a lack of concern with systemic inequalities. Cech (2014) has argued that engineering creates a "culture of disengagement" among engineers, where engineers express a declining interest in public welfare over time. She argued that there are three ideological pillars which contribute to this declining interest in the public good: 1) a social/technical dualism that creates a hierarchical separation between the technical and the social, 2) a belief in individual meritocracy that denies systems of privilege and disadvantage, and 3) a depoliticization of engineering work that frames issues of power and social justice as outside of the engineering field. We argue that efforts to address the first pillar, the social/technical dualism, through reframing engineering as sociotechnical can also address the second two pillars, individual meritocracy and depoliticization, but do not necessarily do so. Many of the ways that "social" aspects have been included in engineering are through a depoliticized approach that limits the potential of engineers to more critically address systemic injustices both internal and external to the engineering field.

Therefore, following Cech's three-pillar premise, we argue that an explicit emphasis on how power and inequality operate within engineering and society is critical. We propose that one way of addressing the limitations of the sociotechnical approaches described above is the explicit inclusion of political engagement and social justice throughout engineering education and practice and in efforts to reframe engineering as a sociotechnical field. This approach addresses 
all three pillars of disengagement in engineering by prompting more critical engagement with how engineering is sociotechnical and questioning how power asymmetries and systemic inequalities affect public welfare throughout engineering education and practice.

Political engagement and social justice are dynamic concepts that can be defined in multiple ways. Social justice is not only "social" in the sense that it affects people, but also that it is socially achieved (Riley 2008), and socially defined (Coordinating Committee of Engineering, Social Justice, and Peace 2010). In this paper, we draw upon a broad definition of social justice developed by the Engineering Social Justice and Peace network, that emphasizes the need to address systemic inequalities in order to develop equitable social conditions (Nieusma 2013). We define political engagement as the explicit consideration of how power shapes social life. Addressing the three pillars would not mean that merit would be insignificant, or that engineering would become partisan, but rather that engineers would pay explicit attention to how power and systemic inequalities affect their profession, practice, and efforts to improve public well-being. Below, we provide conceptual models of sociotechnical approaches both with and without explicit integration of social justice and political engagement in order to demonstrate how this three-pillar approach can be applied.

Model 1: Political engagement and social justice are separate from many sociotechnical frameworks and initiatives in engineering.

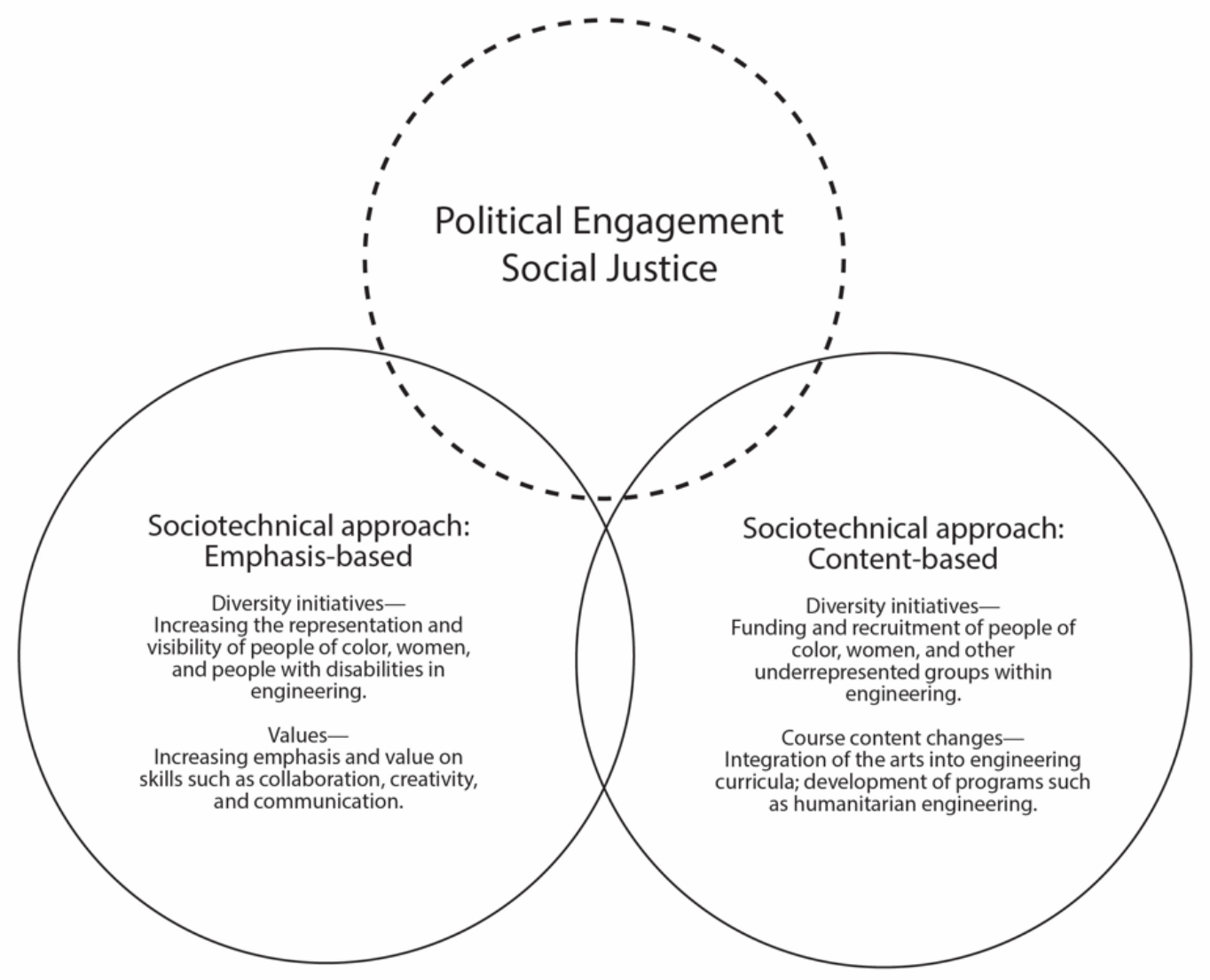




\section{Niles, Roudbari and Contreras}

\section{Integrating Social Justice and Political Engagement into Engineering}

Model 1 represents how "social" issues such as efforts to increase attention to diversity within engineering and efforts to shift curriculum to include arts and humanitarian topics can be integrated into engineering without explicit attention to power and justice. We have elaborated on the limitations of this approach in the section, "Limitations of Dominant Sociotechnical Frameworks."

Model 2: Political engagement and social justice are integrated throughout sociotechnical frameworks and initiatives in engineering.

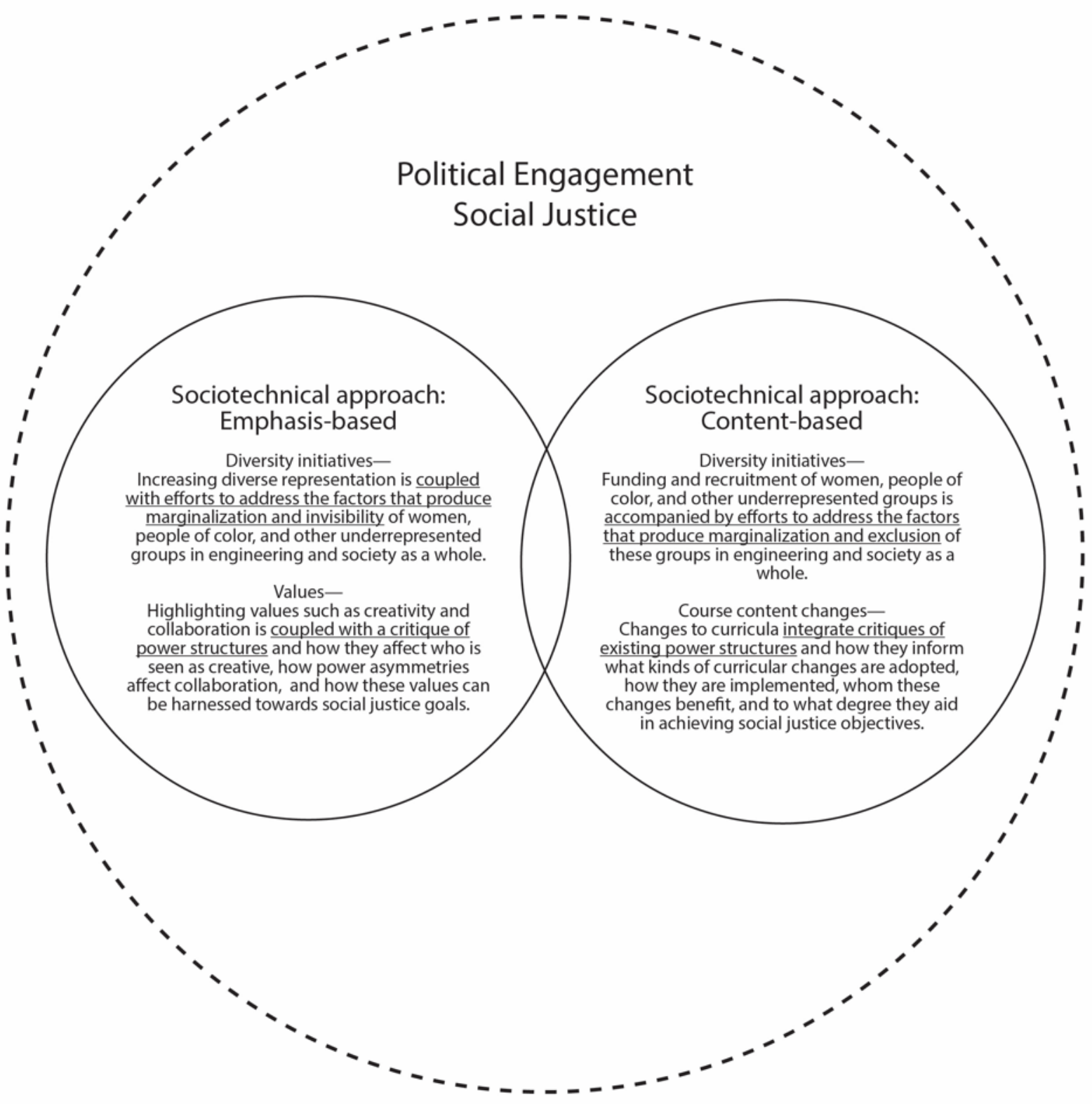

Model 2 demonstrates how political engagement and social justice can be integrated into all sociotechnical frameworks of engineering. In this way, emphasis-based reframings of engineering would also be accompanied by a critique of the systems that produce inequality and marginalize social issues within engineering. Content-based reframings would also involve 
engagement with how new content intersects with and is brought into existing power structures and discriminatory forces within engineering and society. Each of these frameworks for engineering would be oriented towards cultivating knowledge and practices that contribute to social justice goals.

For example, sociotechnical frameworks that emphasize the development of teamwork, empathy, communication, and creativity within engineering education and practice could also be accompanied by a critique of why and how these skills have been devalued in engineering and who gains and who is harmed through the marginalization of these skills. Engineering programs and companies lauding creativity could also ask how what is considered to be "creative" is also shaped by sexism and racism and influenced by the social status of the person or people presenting these ideas. Emphasis on teamwork could include critiques of how power dynamics impact people's ability to contribute to and become valued within a team. The focus on all of these skills could be oriented towards social justice objectives within engineering and society as a whole. For example, considering how engineers can use communication, creativity, and collaboration to better understand and address systemic inequalities.

In addition, expanding engineering education content to include attention to concerns such as global humanitarian issues could include an analysis of how these topics may reinforce the problematic assumption of Western and privileged engineers as experts who are both capable of and justified in intervening in the development of the Global South and in other disadvantaged communities. Furthermore, engineers could also critique how engineering programs may co-opt humanitarian initiatives in order to brand engineering as socially progressive, while at the same time continuing to engage in practices that further harmful military, neoliberal, and extractivist technologies. And, efforts to add new artistic content into engineering education, such as STEAM initiatives, could be integrated with considerations of how diversifying epistemologies in engineering can broaden engineers' abilities to understand and works towards social justice objectives.

Finally, efforts to both highlight existing diversity within engineering and also increase funding and positions available for underrepresented students and faculty could be integrated with widespread efforts to dismantle the structural and cultural factors within engineering and society that marginalize these groups. These are but a few of the ways that integrating explicit attention to political engagement and social justice can be integrated into sociotechnical framings of engineering in order to produce more transformative effects within the engineering field and society as a whole.

\section{Conclusion and Future Directions}

In this article, we describe how engineering can be reframed as sociotechnical in ways that do not necessarily lead to widespread transformations towards social justice, peace, diversity, and improved public welfare. It is possible to bring in questions about social context or values in engineering or highlight the need for more diversity within engineering without broad transformations in the systems that generate social exclusion and harm. We link the limitations of sociotechnical framings of engineering to Cech's three pillars of the "culture of disengagement" in engineering: social/technical dualisms, meritocracy, and depoliticization. We argue that 
reframing engineering as sociotechnical addresses the first pillar, the social/technical dualism, but does not necessarily address the second and third pillars. Therefore, despite important work that engineering scholar-activists have done to advocate for political engagement and emphasis on social justice in engineering, including critiques of militarism, extractivism, capitalism, sexism, and racism, these efforts have not had as transformative of an impact in engineering as many may have hoped. Often, "social" aspects have been brought into engineering in a depoliticized manner that limits the possibilities of engineers to think and act critically towards political and social justice goals.

In order to encompass all three pillars in engineering and to institutionalize a culture within the profession that promotes social justice and peace, we propose that efforts to reframe engineering as a sociotechnical field should be integrated with explicit considerations of political engagement and social justice. Doing so addresses the three pillars of disengagement identified by Cech (2014) which inhibit engineers capacity to serve the public good. Framing engineering as sociotechnical disrupts the first pillar, the social/technical dualism; integrating social justice addresses the second pillar by critiquing the myth of meritocracy and bringing attention to systemic inequalities; and political engagement counters the third pillar of depoliticization bringing attention to how power inequalities shape the engineering profession and practice. Therefore, integrating explicit concerns of political engagement and social justice expands engineers' capacity to contribute to a more just society.

We realize that this is a tall order, and that these are not easy feats. Yet we also believe that naming and describing more just visions for engineering are important actions to take. Furthermore, we recognize that the ways that political engagement and social justice are conceptualized and addressed may vary in different institutional and geographic contexts, including international differences. As a complete assessment of these variations is beyond the scope of this article, we advocate for future scholarship based on empirical studies detailing how political engagement and social justice principles can be applied in a diversity of contexts. We hope that what we have presented here provides greater justification and impetus for engineering students and professionals to explicitly consider how power shapes engineering and society, and how engineering can contribute to reducing social inequalities. Repoliticizing engineering and focusing on systemic inequality and the need for collective responsibility, rather than individual merit, opens up the possibility for engineering to be more broadly transformed into a profession that centers itself on critical service of social justice and peace.

\section{References}

Adams, R., Evangelou, D., English L., de Figueredo, A., Stevens, R., Svinicki, M., Trenor, J., \& Wilson, D. (2011). Multiple perspectives on engaging future engineers. Journal of Engineering Education, 100(1), 48-88. https://doi.org/10.1002/j.21689830.2011.tb00004.x.

American Society of Engineering Education (ASEE) (2018). First bell newsletter. https://www.asee.org/papers-and-publications/blogs-and-newsletters. 
Niles, Roudbari and Contreras

Integrating Social Justice and Political Engagement into Engineering

Benford, R. \& Snow, D. (2000). Framing processes and social movements: An overview and assessment. Annual Review of Sociology, 26, 611-39.

https://doi.org/10.1146/annurev.soc.26.1.611

Bucciarelli, L. (2008). Ethics and engineering education. European Journal of Engineering Education, 33(2), 141-149. https://doi.org/10.1080/03043790801979856.

Cech, E. (2014). Culture of disengagement in engineering education? Science Technology and Human Values, 39(1), 42-72. https://doi.org/10.1177/0162243913504305

Cole, T. (2012). The white savior industrial complex. The Atlantic. March 21, 2012. https://www.theatlantic.com/international/archive/2012/03/the-white-savior-industrialcomplex/254843/

Conlon, E. (2008). The new engineer: Between employability and social responsibility. European Journal of Engineering Education, 33(2), 151-159. https://doi.org/10.1080/03043790801996371

Coordinating Committee of Engineering, Social Justice, and Peace (2010). Our commitments. http://esjp.org/about-esjp/our-commitments

Cumming-Potvin, W., \& Currie, J. (2013). Towards new literacies and social justice for engineering education. International Journal of Engineering, Social Justice, and Peace, 2(1), 21-37. https://doi.org/10.24908/ijesjp.v2i1.3516

Faulkner, W. (2007). "Nuts and bolts and people": Gender-troubled engineering identities. Social Studies of Science, 37(3), 331-356. https://doi.org/10.1177/0306312706072175

Harris, C. (2013) Engineering ethics: From preventive ethics to aspirational ethics. In Michelfelder, D., McCarthy, N., \& Goldberg D. (eds). Philosophy and engineering: Reflections on practice, principles and process, 15, 177-187. Springer. https://doi.org/10.1007/978-94-007-7762-0_14

Herkert, J. (2000). Engineering ethics education in the USA: Content, pedagogy and curriculum. European Journal of Engineering Education, 25(4) 303-313. https://doi.org/10.1080/03043790050200340

Lachman, R. (2018). "STEAM not STEM: Why scientists need arts training." The Conversation. https://theconversation.com/steam-not-stem-why-scientists-need-arts-training-89788

Leong, N. (2013). Racial capitalism. Harvard Law Review, 128(8), 2151-2226. https://doi.org/10.2139/ssrn.2009877

Leydens, J. \& Lucena, J. (2018). Engineering justice: Transforming engineering education and practice. Hoboken, NJ: Wiley-IEEE Press. https://doi.org/10.1002/9781118757369

Lien, T. (2015). "Think you know what an engineer looks like? \#ilooklikeanengineer says think 
Niles, Roudbari and Contreras

Integrating Social Justice and Political Engagement into Engineering

again." Los Angeles Times. August 4, 2015.

https://www.latimes.com/business/technology/la-fi-tn-i-look-like-an-engineer-20150804story.html

Litchfield, K., \& Javernick-Will, A. (2015). "I am an Engineer AND": A mixed methods study of socially engaged engineers. Journal of Engineering Education, 104(4), 393-416. https://doi.org/10.1002/jee.20102

Lynch, W. \& Kline, R. (2000). Engineering practice and engineering ethics. Science, Technology, and Human Values, 25(2), 195-225. https://doi.org/10.1177/016224390002500203

Nieusma, D. (2013). Engineering, social justice, and peace: Strategies for educational and professional reform. In Lucena, J. (ed.), Engineering education for social justice: Critical explorations and ppportunities, 19-40. New York, NY: Springer. https://doi.org/10.1007/97894007-6350-0 2

Nieusma, D. \& Riley, D. (2010). Designs on development: Engineering, globalization, and social justice. Engineering Studies, 2(1), 29-59. https://doi.org/10.1080/19378621003604748

Riley, D. (2008). Engineering and Social Justice. San Rafael, CA: Morgan and Claypool. https://doi.org/10.2200/S00117ED1V01Y200805ETS007

University of Colorado Boulder, College of Engineering and Applied Sciences (n/d). "\#Ilooklikeanengineer." Retrieved September 16, 2019, from https://www.colorado.edu/engineering/ilook

Winner, L. (1980). Do artifacts have politics? Daedalus, 109(1): 121—136. 